\title{
Biharmonic surfaces with parallel mean curvature in complex space forms
}

\author{
Dorel Fetcu and Ana Lucia Pinheiro
}

\begin{abstract}
We classify complete biharmonic surfaces with parallel mean curvature vector field and nonnegative Gaussian curvature in complex space forms.
\end{abstract}

\section{Introduction}

The biharmonic maps were suggested in 1964 by J. Eells and J. H. Sampson as a generalization of harmonic maps (see [9]). Whereas a harmonic map $\varphi$ : $(M, g) \rightarrow(N, h)$ between two Riemannian manifolds is a critical point of the energy functional

$$
E(\varphi)=\frac{1}{2} \int_{M}|d \varphi|^{2} v_{g}
$$

a biharmonic map is a critical point of the bienergy functional

$$
E_{2}(\varphi)=\frac{1}{2} \int_{M}|\tau(\varphi)|^{2} v_{g}
$$

where $\tau(\varphi)=\operatorname{trace} \nabla d \varphi$ is the tension field that vanishes for harmonic maps. The Euler-Lagrange equation corresponding to the bienergy functional was obtained by G. Y. Jiang in 1986 (see [16]):

$$
\begin{aligned}
\tau_{2}(\varphi) & =\Delta \tau(\varphi)-\operatorname{trace} \bar{R}(d \varphi, \tau(\varphi)) d \varphi \\
& =0,
\end{aligned}
$$

where $\tau_{2}(\varphi)$ is the bitension field of $\varphi, \Delta=\operatorname{trace}\left(\nabla^{\varphi}\right)^{2}=\operatorname{trace}\left(\nabla^{\varphi} \nabla^{\varphi}-\nabla_{\nabla}^{\varphi}\right)$ is the rough Laplacian defined on sections of $\varphi^{-1}(T N)$, and $\bar{R}$ is the curvature tensor of $N$, given by $\bar{R}(X, Y) Z=\left[\bar{\nabla}_{X}, \bar{\nabla}_{Y}\right] Z-\bar{\nabla}_{[X, Y]} Z$. Since any harmonic map is biharmonic, we are interested in nonharmonic biharmonic maps, which are called proper-biharmonic.

Kyoto Journal of Mathematics, Vol. 55, No. 4 (2015), 837-855

DOI 10.1215/21562261-3157766, (C) 2015 by Kyoto University

Received July 2, 2014. Revised October 2, 2014. Accepted October 2, 2014.

2010 Mathematics Subject Classification: 53C42, 58E20.

Fetcu's work partially supported by a grant of the Romanian National Authority for Scientific Research (Consiliul National al Cercetarii Stiintifice, Unitatea Executiva pentru Finantarea Invatamantului Superior, a Cercetarii, Dezvoltarii si Inovarii), project number PN-II-RU-TE2011-3-0108. 
A biharmonic submanifold in a Riemannian manifold is a submanifold for which the inclusion map is biharmonic. In Euclidean space the biharmonic submanifolds are the same as those defined by Chen [6], characterized by $\Delta H=0$, where $H$ is the mean curvature vector field and $\Delta$ is the rough Laplacian.

Many classification results for proper-biharmonic submanifolds in space forms, that is, spaces with constant sectional curvature, were obtained in the last decade (see, e.g., [2]-[4]) and the next step was to look for such submanifolds in spaces with nonconstant sectional curvature. Some very important examples of such ambient spaces are the complex space forms, that is, simply connected Kähler manifolds with constant holomorphic sectional curvature. Recent papers such as [12], [13], [21], [24], and [27] treated the subject of proper-biharmonic submanifolds in complex space forms, and several classification results and examples were found.

On the other hand, submanifolds with parallel mean curvature vector (pmc submanifolds) or with constant mean curvature (cmc submanifolds) in Riemannian manifolds proved to be very good candidates for providing nice examples of proper-biharmonic submanifolds (see, e.g., [2]-[4], [14], [22], [27]).

In this article, we first consider pmc surfaces in complex space forms and prove a Simons-type formula for the Laplacian of the squared norm of the holomorphic differential $Q^{(2,0)}$, defined on such a surface, introduced in [11]. Then we use this formula to show that if $\Sigma^{2}$ is a complete pmc surface with nonnegative Gaussian curvature, then the surface is flat or $Q^{(2,0)}$ vanishes on $\Sigma^{2}$. Next, we investigate the biharmonicity of these surfaces, and using a reduction of the codimension theorem of J. H. Eschenburg and R. Tribuzy in [10] and the above-mentioned result, we obtain the following classification theorem.

\section{THEOREM 4.10}

Let $\Sigma^{2}$ be a complete proper-biharmonic pmc surface with nonnegative Gaussian curvature in $\mathbb{C} P^{n}(\rho)$. Then $\Sigma^{2}$ is totally real and one of the following holds.

(a) $\Sigma^{2}$ is pseudo-umbilical and its mean curvature is equal to $\sqrt{\rho} / 2$. Moreover,

$$
\Sigma^{2}=\pi\left(\widetilde{\Sigma}^{2}\right) \subset \mathbb{C} P^{n}(\rho), \quad n \geq 3,
$$

where $\pi: \mathbb{S}^{2 n+1}(\rho / 4) \rightarrow \mathbb{C} P^{n}(\rho)$ is the Hopf fibration and the horizontal lift $\widetilde{\Sigma}^{2}$ of $\Sigma^{2}$ is a complete minimal surface in a small hypersphere $\mathbb{S}^{2 n}(\rho / 2) \subset \mathbb{S}^{2 n+1}(\rho / 4)$.

(b) $\Sigma^{2}$ lies in $\mathbb{C} P^{2}(\rho)$ as a complete Lagrangian proper-biharmonic pmc surface. Moreover, if $\rho=4$, then

$$
\Sigma^{2}=\pi\left(\mathbb{S}^{1}\left(\sqrt{\frac{9 \pm \sqrt{41}}{20}}\right) \times \mathbb{S}^{1}\left(\sqrt{\frac{11 \mp \sqrt{41}}{40}}\right) \times \mathbb{S}^{1}\left(\sqrt{\frac{11 \mp \sqrt{41}}{40}}\right)\right) \subset \mathbb{C} P^{2}(4),
$$

where $\pi: \mathbb{S}^{5}(1) \rightarrow \mathbb{C} P^{2}(4)$ is the Hopf fibration.

(c) $\Sigma^{2}$ lies in $\mathbb{C} P^{3}(\rho)$ and

$$
\Sigma^{2}=\gamma_{1} \times \gamma_{2} \subset \mathbb{C} P^{3}(\rho),
$$


where $\gamma_{1}: \mathbb{R} \rightarrow \mathbb{C} P^{2}(\rho) \subset \mathbb{C} P^{3}(\rho)$ is a holomorphic helix of order 4 with curvatures

$$
\kappa_{1}=\sqrt{\frac{7 \rho}{6}}, \quad \kappa_{2}=\frac{1}{2} \sqrt{\frac{5 \rho}{42}}, \quad \kappa_{3}=\frac{3}{2} \sqrt{\frac{\rho}{42}},
$$

and complex torsions

$$
\tau_{12}=-\tau_{34}=\frac{11 \sqrt{14}}{42}, \quad \tau_{23}=-\tau_{14}=\frac{\sqrt{70}}{42}, \quad \tau_{13}=\tau_{24}=0,
$$

and $\gamma_{2}: \mathbb{R} \rightarrow \mathbb{C} P^{3}(\rho)$ is a holomorphic circle with curvature $\kappa=\sqrt{\rho / 2}$ and complex torsion $\tau_{12}=0$. Moreover, the curves $\gamma_{1}$ and $\gamma_{2}$ always exist and are unique up to holomorphic isometries.

\section{CONVENTIONS}

We work in the $C^{\infty}$-category, which means that manifolds, metrics, connections, and maps are smooth. The Lie algebra of vector fields on a surface $\Sigma^{2}$ is denoted by $C\left(T \Sigma^{2}\right)$. The surfaces are always assumed to be connected and orientable.

\section{Preliminaries}

Let $N^{n}(\rho)$ be a complex space form with complex dimension $n$, complex structure $(J,\langle\cdot, \cdot\rangle)$, and constant holomorphic sectional curvature $\rho$, that is, $N^{n}(\rho)$ is either $\mathbb{C} P^{n}(\rho), \mathbb{C}^{n}$, or $\mathbb{C} H^{n}(\rho)$, according to whether $\rho>0, \rho=0$, or $\rho<0$, respectively. Then the curvature tensor of $(N(\rho), J,\langle\cdot, \cdot\rangle)$ is given by

$$
\begin{aligned}
\bar{R}(X, Y) Z= & \frac{\rho}{4}\{\langle Y, Z\rangle X-\langle X, Z\rangle Y+\langle J Y, Z\rangle J X-\langle J X, Z\rangle J Y \\
& +2\langle X, J Y\rangle J Z\} .
\end{aligned}
$$

Let $\Sigma^{2}$ be a surface immersed in $N^{n}(\rho)$. The second fundamental form $\sigma$ of $\Sigma^{2}$ is defined by the equation of Gauss

$$
\bar{\nabla}_{X} Y=\nabla_{X} Y+\sigma(X, Y)
$$

while the shape operator $A$ and the normal connection $\nabla^{\perp}$ are given by the equation of Weingarten

$$
\bar{\nabla}_{X} V=-A_{V} X+\nabla_{X}^{\perp} V,
$$

for any vector fields $X$ and $Y$ tangent to the surface and any vector field $V$ normal to $\Sigma^{2}$, where $\bar{\nabla}$ and $\nabla$ are the Levi-Civita connections of $N^{n}(\rho)$ and $\Sigma^{2}$, respectively.

\section{DEFINITION 2.1}

If the mean curvature vector $H$ of the surface $\Sigma^{2}$ is parallel in the normal bundle, that is, $\nabla^{\perp} H=0$, then $\Sigma^{2}$ is called a pmc surface.

We end this section by recalling some notions and results from the theory of Frenet curves in complex space forms, which we shall use later. Let $\gamma: I \subset \mathbb{R} \rightarrow$ 
$N^{n}(\rho)$ be a curve parameterized by arc length. Then $\gamma$ is called a Frenet curve of osculating order $r, 1 \leq r \leq 2 n$, if there exist $r$ orthonormal vector fields $\left\{X_{1}=\right.$ $\left.\gamma^{\prime}, \ldots, X_{r}\right\}$ along $\gamma$ such that

$$
\begin{aligned}
& \bar{\nabla}_{X_{1}} X_{1}=\kappa_{1} X_{2}, \quad \bar{\nabla}_{X_{1}} X_{i}=-\kappa_{i-1} X_{i-1}+\kappa_{i} X_{i+1}, \quad \ldots, \\
& \bar{\nabla}_{X_{1}} X_{r}=-\kappa_{r-1} X_{r-1},
\end{aligned}
$$

for all $i \in\{2, \ldots, r-1\}$, where $\left\{\kappa_{1}, \kappa_{2}, \ldots, \kappa_{r-1}\right\}$ are positive functions on $I$ called the curvatures of $\gamma$. These equations are called the Frenet equations of $\gamma$.

A Frenet curve of osculating order $r$ is called a helix of order $r$ if $\kappa_{i}=$ const $>$ 0 for $1 \leq i \leq r-1$. A helix of order 2 is called a circle, and a helix of order 3 is simply called a helix.

S. Maeda and Y. Ohnita [20] defined the complex torsions $\tau_{i j}$ of the curve $\gamma$ by $\tau_{i j}=\left\langle X_{i}, J X_{j}\right\rangle$, where $1 \leq i<j \leq r$. A helix of order $r$ is called a holomorphic helix of order $r$ if all its complex torsions are constant. We note that a circle is always a holomorphic circle.

In [19] the following existence results are proved.

\section{THEOREM 2.2 ([19])}

For given positive constants $\kappa_{1}, \kappa_{2}$, and $\kappa_{3}$, there exist four equivalence classes of holomorphic helices of order 4 in $\mathbb{C} P^{2}(\rho)$ with curvatures $\kappa_{1}, \kappa_{2}$, and $\kappa_{3}$ with respect to holomorphic isometries of $\mathbb{C} P^{2}(\rho)$. The four classes are defined by certain relations on the complex torsions and they are as follows. When $\kappa_{1} \neq \kappa_{3}$,

$$
\begin{aligned}
& I_{1}: \quad \tau_{12}=\tau_{34}=\mu, \quad \tau_{23}=\tau_{14}=\kappa_{2} \mu /\left(\kappa_{1}+\kappa_{3}\right), \quad \tau_{13}=\tau_{24}=0, \\
& I_{2}: \quad \tau_{12}=\tau_{34}=-\mu, \quad \tau_{23}=\tau_{14}=-\kappa_{2} \mu /\left(\kappa_{1}+\kappa_{3}\right), \quad \tau_{13}=\tau_{24}=0, \\
& I_{3}: \quad \tau_{12}=-\tau_{34}=\nu, \quad \tau_{23}=-\tau_{14}=\kappa_{2} \nu /\left(\kappa_{1}-\kappa_{3}\right), \quad \tau_{13}=\tau_{24}=0, \\
& I_{4}: \quad \tau_{12}=-\tau_{34}=-\nu, \quad \tau_{23}=-\tau_{14}=-\kappa_{2} \nu /\left(\kappa_{1}-\kappa_{3}\right), \quad \tau_{13}=\tau_{24}=0,
\end{aligned}
$$

where

$$
\mu=\frac{\kappa_{1}+\kappa_{3}}{\sqrt{\kappa_{2}^{2}+\left(\kappa_{1}+\kappa_{3}\right)^{2}}} \quad \text { and } \quad \nu=\frac{\kappa_{1}-\kappa_{3}}{\sqrt{\kappa_{2}^{2}+\left(\kappa_{1}-\kappa_{3}\right)^{2}}},
$$

and when $\kappa_{1}=\kappa_{3}$ the classes $I_{3}$ and $I_{4}$ are substituted by

$$
\begin{aligned}
& I_{3}^{\prime}: \quad \tau_{12}=\tau_{34}=\tau_{13}=\tau_{24}=0, \quad \tau_{23}=-\tau_{14}=1, \\
& I_{4}^{\prime}: \quad \tau_{12}=\tau_{34}=\tau_{13}=\tau_{24}=0, \quad \tau_{23}=-\tau_{14}=-1 .
\end{aligned}
$$

\section{THEOREM 2.3 ([19])}

For any positive number $\kappa$ and for any number $\tau$ such that $|\tau|<1$, there exists a holomorphic circle with curvature $\kappa$ and complex torsion $\tau$ in any complex space form. 


\section{A Simons-type formula for pmc surfaces in complex space forms}

Let $\left(N^{n}(\rho), J,\langle\cdot, \cdot\rangle\right)$ be a complex space form, with constant holomorphic sectional curvature $\rho$ and complex dimension $n$, and let $\Sigma^{2}$ be a pmc surface in $N^{n}(\rho)$.

In [11] it is proved that the $(2,0)$-part $Q^{(2,0)}$ of the quadratic form $Q$ defined on $\Sigma^{2}$ by

$$
Q(X, Y)=8|H|^{2}\left\langle A_{H} X, Y\right\rangle+3 \rho\langle X, T\rangle\langle Y, T\rangle,
$$

where $T$ is the tangent part $(J H)^{\top}$ of $J H$, is holomorphic.

Using this holomorphic differential we shall prove the following result.

\section{THEOREM 3.1}

Let $\Sigma^{2}$ be a complete nonminimal pmc surface with nonnegative Gaussian curvature $K$ isometrically immersed in a complex space form $N^{n}(\rho), \rho \neq 0$. Then one of the following holds:

(a) the surface is flat; or

(b) there exists a point $p \in \Sigma^{2}$ such that $K(p)>0$ and $Q^{(2,0)}$ vanishes on $\Sigma^{2}$.

Proof

First, we recall a Simons-type equation obtained by S.-Y. Cheng and S.-T. Yau $[8,(2.8)]$, which generalizes a result of J. Simons [26]. Let $M$ be an $m$-dimensional Riemannian manifold, and consider a symmetric operator $S$ on $M$ that satisfies the Codazzi equation $\left(\nabla_{X} S\right) Y=\left(\nabla_{Y} S\right) X$, where $\nabla$ is the Levi-Civita connection on the manifold. Then, we have that

$$
\frac{1}{2} \Delta|S|^{2}=|\nabla S|^{2}+\sum_{i=1}^{m} \lambda_{i}(\operatorname{trace} S)_{i i}+\frac{1}{2} \sum_{i, j=1}^{m} R_{i j i j}\left(\lambda_{i}-\lambda_{j}\right)^{2},
$$

where $\lambda_{i}, 1 \leq i \leq m$, are the eigenvalues of $S$, and the $R_{i j k l}$ 's are the components of the Riemannian curvature of $M$.

Next, let us consider the following operator $S$, defined on our surface $\Sigma^{2}$ by

$$
S=8|H|^{2} A_{H}+3 \rho\langle T, \cdot\rangle T-\left(\frac{3 \rho}{2}|T|^{2}+8|H|^{4}\right) \mathrm{I} .
$$

We shall prove that $|S|^{2}$ is a bounded subharmonic function on the surface.

First, it is easy to see that

$$
\langle S X, Y\rangle=Q(X, Y)-\frac{\operatorname{trace} Q}{2}\langle X, Y\rangle,
$$

which implies that $S$ is symmetric and traceless. It is also easy to see that $Q^{(2,0)}$ vanishes on $\Sigma^{2}$ if and only if $S=0$ on the surface.

Using (3.4), since $Q^{(2,0)}$ is holomorphic, just as in [5, Proposition 3.3] one can prove that $S$ satisfies the Codazzi equation $\left(\nabla_{X} S\right) Y=\left(\nabla_{Y} S\right) X$, where $\nabla$ is the Levi-Civita connection on the surface. Then, from (3.2) and the fact that 
trace $S=0$, we easily get that

$$
\frac{1}{2} \Delta|S|^{2}=2 K|S|^{2}+|\nabla S|^{2},
$$

where $K$ is the Gaussian curvature of the surface.

Now, let us consider the local orthonormal frame field $\left\{E_{3}=H /|H|, E_{4}\right.$, $\left.\ldots, E_{2 n}\right\}$ in the normal bundle, and denote $A_{\alpha}=A_{E_{\alpha}}$. It follows that trace $A_{3}=$ $2|H|$ and trace $A_{\alpha}=0$, for all $\alpha>3$.

From the definition (3.3) of $S$, we have, after a straightforward computation, that

$$
\operatorname{det} A_{3}=\frac{1}{|H|^{2}} \operatorname{det} A_{H}=|H|^{2}-\frac{1}{128|H|^{6}}|S|^{2}-\frac{9 \rho^{2}}{256|H|^{6}}|T|^{4}+\frac{3 \rho}{64|H|^{6}}\langle S T, T\rangle,
$$

and then, by using the equation of Gauss for $\Sigma^{2}$ in $N$,

$$
\begin{aligned}
R(X, Y) Z= & \frac{\rho}{4}\{\langle Y, Z\rangle X-\langle X, Z\rangle Y+\langle J Y, Z\rangle J X-\langle J X, Z\rangle J Y \\
& +2\langle X, J Y\rangle J Z\}+\sum_{\alpha=3}^{2 n}\left\{\left\langle A_{\alpha} Y, Z\right\rangle A_{\alpha} X-\left\langle A_{\alpha} X, Z\right\rangle A_{\alpha} Y\right\},
\end{aligned}
$$

the Gaussian curvature can be written as

$$
\begin{aligned}
K= & \frac{\rho}{4}\left(1+3 \cos ^{2} \theta\right)+|H|^{2}-\frac{1}{128|H|^{6}}|S|^{2}-\frac{9 \rho^{2}}{256|H|^{6}}|T|^{4} \\
& +\frac{3 \rho}{64|H|^{6}}\langle S T, T\rangle+\sum_{\alpha>3} \operatorname{det} A_{\alpha},
\end{aligned}
$$

where $\theta=\left\langle J E_{1}, E_{2}\right\rangle$ is the Kähler angle function of $\Sigma^{2},\left\{E_{1}, E_{2}\right\}$ being a local orthonormal positively oriented frame field in the tangent bundle.

Since trace $A_{\alpha}=0$, it follows that $\operatorname{det} A_{\alpha} \leq 0$, for all $\alpha>3$. Therefore, as $K \geq 0$, we get the following global formula:

$$
-\frac{1}{128|H|^{2}}|S|^{2}+\frac{3 \rho}{64|H|^{6}}\langle S T, T\rangle-\frac{9 \rho^{2}}{256|H|^{6}}|T|^{4}+|H|^{2}+\frac{\rho}{4}\left(1+3 \cos ^{2} \theta\right) \geq 0 .
$$

From $|\langle S T, T\rangle| \leq(1 / \sqrt{2})|T||S|$, since $|T| \leq|J H|=|H|$, we have that $\rho\langle S T, T\rangle \leq$ $(|\rho| / \sqrt{2})|H|^{2}|S|$, which implies that

$$
-\frac{1}{128|H|^{6}}|S|^{2}+\frac{3|\rho|}{64 \sqrt{2}|H|^{4}}|S|+|H|^{2}+\frac{\rho}{4}\left(1+3 \cos ^{2} \theta\right) \geq 0 .
$$

In the following we shall prove that $|S|$ is bounded. We have two cases: $\rho<0$ or $\rho>0$. If $\rho<0$, then we have that

$$
-\frac{1}{128|H|^{6}}|S|^{2}+\frac{3 \rho}{64 \sqrt{2}|H|^{4}}|S|+|H|^{2} \geq 0
$$

and then $|S| \leq\left(\sqrt{9 \rho^{2}+256|H|^{4}}-3 \rho\right)|H|^{2} / \sqrt{2}$. When $\rho>0$, we get that

$$
-\frac{1}{128|H|^{6}}|S|^{2}+\frac{3 \rho}{64 \sqrt{2}|H|^{4}}|S|+|H|^{2}+\rho \geq 0,
$$

which is equivalent to $|S| \leq\left(\sqrt{9 \rho^{2}+256 \rho|H|^{2}+256|H|^{4}}+3 \rho\right)|H|^{2} / \sqrt{2}$. 
Since the surface is complete and has nonnegative Gaussian curvature, we see, using a result of A. Huber [15], that $\Sigma^{2}$ is a parabolic space. From the above calculation and (3.5), we get that $|S|^{2}$ is a bounded subharmonic function, which implies that $|S|$ is a constant. Then, from (3.5), we get that $K=0$ on $\Sigma^{2}$ or there exists a point $p \in \Sigma^{2}$ such that $K(p)>0$ and then $S=0$ on the surface, which, as we have seen, is equivalent to $Q^{(2,0)}=0$.

REMARK 3.2

For a surface $\Sigma^{2}$ as in Theorem 3.1 we have $|S|=$ const and $\nabla S=0$.

\section{Biharmonic pmc surfaces in $\mathbb{C} P^{n}(\rho)$}

To prove our main result we shall need the following theorem.

THEOREM 4.1 ([3])

A submanifold $\Sigma^{m}$ in a Riemannian manifold $N$, with second fundamental form $\sigma$, mean curvature vector field $H$, and shape operator $A$, is biharmonic if and only if

$$
\left\{\begin{array}{l}
-\Delta^{\perp} H+\operatorname{trace} \sigma\left(\cdot, A_{H} \cdot\right)+\operatorname{trace}(\bar{R}(\cdot, H) \cdot)^{\perp}=0, \\
\frac{m}{2} \operatorname{grad}|H|^{2}+2 \operatorname{trace} A_{\nabla^{\perp} H}(\cdot)+2 \operatorname{trace}(\bar{R}(\cdot, H) \cdot)^{\top}=0,
\end{array}\right.
$$

where $\Delta^{\perp}$ is the Laplacian in the normal bundle and $\bar{R}$ is the curvature tensor of $N$.

Using the formula (2.1) of the curvature tensor of a complex space form $N(\rho)$, we get the following result.

\section{COROLLARY 4.2}

Let $\Sigma^{2}$ be a pmc surface in a complex space form $(N(\rho), J,\langle\cdot, \cdot\rangle)$. Then $\Sigma^{2}$ is biharmonic if and only if

$$
\operatorname{trace} \sigma\left(\cdot, A_{H} \cdot\right)=\frac{\rho}{4}\left\{2 H-3(J T)^{\perp}\right\} \quad \text { and } \quad(J T)^{\top}=0,
$$

where $T$ is the tangent part of $J H$ and $(J T)^{\perp}$ and $(J T)^{\top}$ are the normal and the tangent part of $J T$, respectively.

\section{REMARK 4.3}

It is easy to see, from the first equation of (4.1), that for a proper-biharmonic pmc surface

$$
0<\left|A_{H}\right|^{2}=\frac{\rho}{4}\left\{2|H|^{2}+3|T|^{2}\right\},
$$

which implies that $\rho>0$, and therefore, such surfaces exist only in $\mathbb{C} P^{n}(\rho)$.

PROPOSITION 4.4

If $\Sigma^{2}$ is a proper-biharmonic pmc surface in $\mathbb{C} P^{n}(\rho)$, then $T$ has constant length. 


\section{Proof}

The map $p \in \Sigma^{2} \rightarrow\left(A_{H}-\mu \mathrm{I}\right)(p)$, where $\mu$ is a constant, is analytic, and therefore, either $\Sigma^{2}$ is a pseudo-umbilical surface (at every point), or $H$ is an umbilical direction on a closed set without interior points (see [1], [11]). We shall denote by $W$ the set of points where $H$ is not an umbilical direction. Since in the second case this set is open and dense in $\Sigma^{2}$, when the surface is not pseudo-umbilical we shall work on $W$ and then extend our results throughout $\Sigma^{2}$ by continuity. If $\Sigma^{2}$ is pseudo-umbilical, then $J H$ is normal to the surface, that is, $T=0$ on the surface (see [25]).

Let us assume now that $\Sigma^{2}$ is not pseudo-umbilical, and let $N$ be the normal part of $J H$. Then, for any vector field $X$ tangent to the surface, we have that

$$
\begin{aligned}
\bar{\nabla}_{X} J H & =-J \bar{\nabla}_{X} H=-J A_{H} X \\
& =\nabla_{X} T+\sigma(X, T)-A_{N} X+\nabla_{X}^{\perp} N
\end{aligned}
$$

and, therefore,

$$
\left\langle\nabla_{X} T, T\right\rangle=\left\langle A_{N} X, T\right\rangle+\left\langle A_{H} X, J T\right\rangle=\left\langle A_{N} X, T\right\rangle,
$$

since, from the second equation of (4.1), we know that $J T$ is normal.

It is easy to see that

$$
\langle N, H\rangle=0 \quad \text { and } \quad\langle N, J T\rangle=0
$$

and, again by using (4.1), that

$$
\langle N, J X\rangle=0, \quad \forall X \in C\left(T \Sigma^{2}\right) .
$$

Then, from the first equation of (4.1), we get that

$$
\operatorname{trace}\left(A_{H} A_{N}\right)=0 \text {. }
$$

Moreover, using the Ricci equation

$$
\left\langle R^{\perp}(X, Y) H, V\right\rangle=\left\langle\left[A_{H}, A_{V}\right] X, Y\right\rangle+\langle\bar{R}(X, Y) H, V\rangle, \quad \forall V \in C\left(N \Sigma^{2}\right),
$$

we obtain that

$$
\left[A_{H}, A_{N}\right] T=0,
$$

since $R^{\perp}(X, Y) H=0$ and $\langle\bar{R}(X, T) H, N\rangle=0$, for tangent vector fields $X$ and $Y$.

Next, consider a point $p \in W$ and an orthonormal basis $\left\{e_{1}, e_{2}\right\}$ in $T_{p} \Sigma^{2}$ such that $A_{H} e_{i}=\lambda_{i} e_{i}, i \in\{1,2\}$. Obviously, we have that $\lambda_{1} \neq \lambda_{2}$ and we can write $A_{H}$ and $A_{N}$ with respect to $\left\{e_{1}, e_{2}\right\}$ as

$$
A_{H}=\left(\begin{array}{cc}
\lambda_{1} & 0 \\
0 & \lambda_{2}
\end{array}\right) \quad \text { and } \quad A_{N}=\left(\begin{array}{cc}
a & b \\
b & -a
\end{array}\right),
$$

since $N \perp H$, that is, trace $A_{N}=0$. From (4.3) we get $a=0$ and then (4.5) becomes

$$
\left(\lambda_{2}-\lambda_{1}\right) b\left(\left\langle T, e_{2}\right\rangle e_{1}-\left\langle T, e_{1}\right\rangle e_{2}\right)=0 .
$$


Therefore, at $p$, we have that either $T=0$ or $b=0$. We can see that in both cases $\left\langle A_{N} X, T\right\rangle=0$, which implies that (4.2) reduces to

$$
X\left(|T|^{2}\right)=2\left\langle\nabla_{X} T, T\right\rangle=0
$$

for any tangent vector $X$. It follows that $X\left(|T|^{2}\right)=0$ for any tangent vector field $X$ on $\Sigma^{2}$, which means that $|T|$ is constant on the surface.

\section{REMARK 4.5}

If $|T|=$ const $\neq 0$, then we have that $\nabla_{X} T=A_{N} X=0$ for any tangent vector field $X$. Indeed, if $T \neq 0$ everywhere, then since $J T$ is a normal vector field, it follows that $\Sigma^{2}$ is a totally real surface. Then we get that

$$
\bar{\nabla}_{X} J H=-J \bar{\nabla}_{X} H=-J A_{H} X \in C\left(N \Sigma^{2}\right),
$$

which means that $\nabla_{X} T=A_{N} X$. On the other hand, we have that $\langle\bar{R}(X, Y) H$, $N\rangle=0$ for any tangent vector fields $X$ and $Y$, and then, from the Ricci equation (4.4), one sees that $\left[A_{H}, A_{N}\right]=0$. Using this equation and (4.3) in the same way as in the proof of Proposition 4.4 and since $T \neq 0$ implies that $H$ is not umbilical on an open dense set, we obtain that $A_{N}=0$ on this set and, therefore, on the whole surface.

\section{PROPOSITION 4.6}

If $\Sigma^{2}$ is a complete proper-biharmonic pmc surface in $\mathbb{C} P^{n}(\rho)$ with nonnegative Gaussian curvature $K$ and $T=0$, then $n \geq 3$ and $\Sigma^{2}$ is pseudo-umbilical and totally real. Moreover, the mean curvature of $\Sigma^{2}$ is $|H|=\sqrt{\rho} / 2$.

Proof

From Corollary 4.2 we see that the pmc surface $\Sigma^{2}$ with $T=0$ is properbiharmonic if and only if

$$
\operatorname{trace} \sigma\left(\cdot, A_{H} \cdot\right)=\frac{\rho}{2} H
$$

Now, from Theorem 3.1, we know that either the Gaussian curvature $K$ vanishes identically on the surface, or there exists a point $p \in \Sigma^{2}$ such that $K(p)>0$ and $Q^{(2,0)}=0$ on $\Sigma^{2}$.

In the second case, since $T=0$ and $Q^{(2,0)}=0$, it is easy to see that $\Sigma^{2}$ is pseudo-umbilical and then totally real (see [25]). From (4.6), we get that $\left|A_{H}\right|^{2}=$ $(\rho / 2)|H|^{2}$, but since $\Sigma^{2}$ is pseudo-umbilical, we also have that $\left|A_{H}\right|^{2}=2|H|^{4}$, which means that $|H|=\sqrt{\rho} / 2$.

If the surface is flat, then we shall prove first that it is also totally real. Since $J H$ is a normal vector field to $\Sigma^{2}$, we have that

$$
\begin{aligned}
\bar{\nabla}_{X} J H & =J \bar{\nabla}_{X} H=-J A_{H} X \\
& =-A_{J H} X+\nabla_{X}^{\perp} J H .
\end{aligned}
$$

Let us now consider an orthonormal basis $\left\{e_{1}, e_{2}\right\}$ in $T_{p} \Sigma^{2}$, where $p \in \Sigma^{2}$, such that $A_{H} e_{i}=\lambda_{i} e_{i}, i \in\{1,2\}$. It follows that $J A_{H} e_{i}=\lambda_{i} J e_{i}$, and for $i \neq j$, 
we have that

$$
\left\langle A_{J H} e_{i}, e_{j}\right\rangle=\left\langle J A_{H} e_{i}, e_{j}\right\rangle=\lambda_{i}\left\langle J e_{i}, e_{j}\right\rangle .
$$

Thus, we obtained that $\lambda_{1}\left\langle J e_{1}, e_{2}\right\rangle=\lambda_{2}\left\langle J e_{2}, e_{1}\right\rangle$, which means that

$$
0=\left(\lambda_{1}+\lambda_{2}\right)\left\langle J e_{1}, e_{2}\right\rangle=2|H|^{2}\left\langle J e_{1}, e_{2}\right\rangle .
$$

Therefore, we have that $\left\langle J e_{1}, e_{2}\right\rangle=0$; that is, $\Sigma^{2}$ is totally real.

In the following, we will prove that $\Sigma^{2}$ is also pseudo-umbilical. Assume that it is not so, and we will work on the set $W$ defined in the proof of Proposition 4.4. Let $p$ be a point in $W$, consider a basis $\left\{e_{1}, e_{2}\right\}$ in $T_{p} \Sigma^{2}$ such that $A_{H} e_{i}=\lambda_{i} e_{i}$, and extend the $e_{i}$ 's to vector fields $E_{i}$ in a neighborhood of $p$. First, using the expression (2.1) of the curvature tensor of $\mathbb{C} P^{n}(\rho)$, we obtain that $\left\langle\bar{R}\left(E_{2}, E_{1}\right) H, J E_{1}\right\rangle=0$ and then, from the Ricci equation (4.4), $\left\langle\left[A_{H}, A_{J E_{1}}\right] E_{1}\right.$, $\left.E_{2}\right\rangle=0$, which can be written at $p$ as

$$
\left(\lambda_{2}-\lambda_{1}\right)\left\langle A_{J E_{1}} E_{1}, E_{2}\right\rangle=0 .
$$

In the same way, we can also show that $\left(\lambda_{1}-\lambda_{2}\right)\left\langle A_{J E_{2}} E_{2}, E_{1}\right\rangle=0$.

First, since $\lambda_{1} \neq \lambda_{2}$, we get that $\left\langle A_{J E_{1}} E_{1}, E_{2}\right\rangle=\left\langle A_{J E_{2}} E_{2}, E_{1}\right\rangle=0$. Using the fact that $\Sigma^{2}$ is totally real, it is easy to verify that

$$
\langle\sigma(X, Y), J Z\rangle=\langle\sigma(X, Z), J Y\rangle, \quad \forall X, Y, Z \in C\left(T \Sigma^{2}\right),
$$

and then, at $p$, we obtain that

$$
\left\langle A_{J E_{2}} E_{1}, E_{1}\right\rangle=\left\langle\sigma\left(E_{1}, E_{1}\right), J E_{2}\right\rangle=\left\langle\sigma\left(E_{1}, E_{2}\right), J E_{1}\right\rangle=\left\langle A_{J E_{1}} E_{1}, E_{2}\right\rangle=0
$$

and

$$
\left\langle A_{J E_{1}} E_{2}, E_{2}\right\rangle=\left\langle A_{J E_{2}} E_{2}, E_{1}\right\rangle=0 .
$$

Since $J H$ being normal to $\Sigma^{2}$ is equivalent to $\operatorname{trace} A_{J E_{1}}=\operatorname{trace} A_{J E_{2}}=0$, we have just proved that $A_{J E_{1}}=A_{J E_{2}}=0$ at $p$.

Next, for any normal vector field $U$ which is also orthogonal to $H, J E_{1}$, and $J E_{2}$, we have that $\langle\bar{R}(X, Y) H, U\rangle=0$ and then, from the Ricci equation (4.4), $\left[A_{H}, A_{U}\right]=0$. Since $H$ is not umbilical on $W$, this implies that, with respect to $\left\{E_{1}, E_{2}\right\}$, at $p$,

$$
A_{H}=\left(\begin{array}{cc}
a+|H|^{2} & 0 \\
0 & -a+|H|^{2}
\end{array}\right) \quad \text { and } \quad A_{U}=\left(\begin{array}{cc}
b & 0 \\
0 & -b
\end{array}\right),
$$

with $a \neq 0$. From (4.6) we have that $\operatorname{trace}\left(A_{H} A_{U}\right)=0$, which implies, using the above expressions, that $A_{U}=0$.

Now, we consider a local orthonormal frame field in the normal bundle of $\Sigma^{2}$ as follows: $\left\{E_{3}=H /|H|, E_{4}=J E_{1}, E_{5}=J E_{2}, E_{6}, \ldots, E_{2 n}\right\}$. Since the surface is flat, by the Gauss equation (3.6) of $\Sigma^{2}$ in $\mathbb{C} P^{n}(\rho)$, at $p$ we get that

$$
0=K=\frac{\rho}{4}+\sum_{\alpha=3}^{2 n} \operatorname{det} A_{\alpha}=\frac{\rho}{4}+\operatorname{det} A_{3}=\frac{\rho}{4}+|H|^{2}-\frac{a^{2}}{|H|^{2}} .
$$


From (4.6) we have that $\left|A_{H}\right|^{2}=2 a^{2}+2|H|^{4}=(\rho / 2)|H|^{2}$ and, therefore, $K=$ $2|H|^{2}$ at $p$, which means that $|H|=0$. This is a contradiction, since $\Sigma^{2}$ is proper biharmonic. Hence, the surface is pseudo-umbilical in this case too.

Finally, we have that, for any vector field $X$ tangent to the surface, the vector field $J X$ is normal and orthogonal to both $H$ and $J H$, which are also normal vector fields. Therefore, one obtains that $n \geq 3$ and we conclude.

\section{PROPOSITION 4.7}

If $\Sigma^{2}$ is a complete proper-biharmonic pmc surface in $\mathbb{C} P^{n}(\rho)$ with nonnegative Gaussian curvature $K$ and $T \neq 0$, then the surface is flat and $\nabla A_{H}=0$.

\section{Proof}

Since $|T|=$ const $\neq 0$ on $\Sigma^{2}$, from the second equation of (4.1), we know that our surface is totally real. In [7] it is proved that the $(2,0)$-part $\widetilde{Q}^{(2,0)}$ of the quadratic form

$$
\widetilde{Q}(X, Y)=\left\langle A_{H} X, Y\right\rangle
$$

defined on a pmc totally real surface, is holomorphic. Consider the traceless part $\phi_{H}=A_{H}-|H|^{2} \mathrm{I}$ of $A_{H}$. Since $\widetilde{Q}^{(2,0)}$ is holomorphic, working in the same way as in [5, Proposition 3.3], we can prove that $\phi_{H}$ satisfies the Codazzi equation $\left(\nabla_{X} \varnothing_{H}\right) Y=\left(\nabla_{Y} \varnothing_{H}\right) X$. Hence, from (3.2), we have that

$$
\frac{1}{2} \Delta\left|\phi_{H}\right|^{2}=2 K\left|\phi_{H}\right|^{2}+\left|\nabla \phi_{H}\right|^{2} .
$$

Let us assume now that there exists a point $p \in \Sigma^{2}$ such that $K(p)>0$. Then, from Theorem 3.1, we have that $S=0$, which implies that

$$
\left|\phi_{H}\right|^{2}=\left|A_{H}\right|^{2}-2|H|^{4}=\frac{9 \rho^{2}}{128|H|^{4}}|T|^{4}=\text { const } \neq 0,
$$

which means that $K=0$ on $\Sigma^{2}$. This is a contradiction.

Hence, the surface is flat. Since $\Sigma^{2}$ is proper-biharmonic, it follows, from the first equation of (4.1), that $\left|\phi_{H}\right|^{2}$ is bounded. Thus, $\left|\phi_{H}\right|^{2}$ is a bounded subharmonic function on a parabolic space and, therefore, a constant, which implies that $\nabla A_{H}=\nabla \phi_{H}=0$.

\section{REMARK 4.8}

In the proof of Proposition 4.7 we used the fact that $\widetilde{Q}^{(2,0)}$ is holomorphic when $\widetilde{Q}$ is defined on a totally real pmc surface in a complex space form. In [18] it is proved that if $\Sigma^{2}$ is a proper-biharmonic surface with constant mean curvature in a Riemannian manifold, then $\widetilde{Q}^{(2,0)}$ is holomorphic.

Before proving our main result, let us briefly recall a property of the Hopf fibration (see [23]). Let $\pi: \mathbb{C}^{n+1} \backslash\{0\} \rightarrow \mathbb{C} P^{n}(\rho)$ be the natural projection, and let $\mathbb{S}^{2 n+1}(\rho / 4)=\left\{z \in \mathbb{C}^{n+1}:\langle z, z\rangle=4 / \rho\right\}$. The restriction of $\pi$ to the sphere $\mathbb{S}^{2 n+1}(\rho / 4) \subset \mathbb{C}^{n+1}$ is the Hopf fibration $\pi: \mathbb{S}^{2 n+1}(\rho / 4) \rightarrow \mathbb{C} P^{n}(\rho)$ and it is a 
Riemannian submersion. Now, let $i: \Sigma^{m} \rightarrow \mathbb{C} P^{n}(\rho)$ be a totally real isometric immersion. Then this immersion can be lifted locally (or globally, if $\Sigma^{m}$ is simply connected) to a horizontal immersion $\widetilde{i}: \widetilde{\Sigma}^{m} \rightarrow \mathbb{S}^{2 n+1}(\rho / 4)$. Conversely, if $\widetilde{i}$ : $\widetilde{\Sigma}^{m} \rightarrow \mathbb{S}^{2 n+1}(\rho / 4)$ is a horizontal isometric immersion, then $\pi(\widetilde{i}): \Sigma^{m} \rightarrow \mathbb{C} P^{n}(\rho)$ is a totally real isometric immersion. Moreover, we have that $\pi_{*} \widetilde{\sigma}=\sigma$, where $\widetilde{\sigma}$ and $\sigma$ are the second fundamental forms of the immersions $\widetilde{i}$ and $i$, respectively. We shall also use the following theorem.

\section{THEOREM 4.9 ([2])}

Let $\Sigma^{m}$ be a proper-biharmonic cmc submanifold in $\mathbb{S}^{n}(\rho / 4)$ with mean curvature vector field $H$. Then $|H| \in(0, \sqrt{\rho} / 2]$ and, moreover, $|H|=\sqrt{\rho} / 2$ if and only if $\Sigma^{m}$ is minimal in a small hypersphere $\mathbb{S}^{n-1}(\rho / 2) \subset \mathbb{S}^{n}(\rho / 4)$.

We are now ready to prove our main result.

\section{THEOREM 4.10}

Let $\Sigma^{2}$ be a complete proper-biharmonic pmc surface with nonnegative Gaussian curvature in $\mathbb{C} P^{n}(\rho)$. Then $\Sigma^{2}$ is totally real, and one of the following holds.

(a) $\Sigma^{2}$ is pseudo-umbilical and its mean curvature is equal to $\sqrt{\rho} / 2$. Moreover,

$$
\Sigma^{2}=\pi\left(\widetilde{\Sigma}^{2}\right) \subset \mathbb{C} P^{n}(\rho), \quad n \geq 3,
$$

where $\pi: \mathbb{S}^{2 n+1}(\rho / 4) \rightarrow \mathbb{C} P^{n}(\rho)$ is the Hopf fibration and the horizontal lift $\widetilde{\Sigma}^{2}$ of $\Sigma^{2}$ is a complete minimal surface in a small hypersphere $\mathbb{S}^{2 n}(\rho / 2) \subset \mathbb{S}^{2 n+1}(\rho / 4)$.

(b) $\Sigma^{2}$ lies in $\mathbb{C} P^{2}(\rho)$ as a complete Lagrangian proper-biharmonic pmc surface. Moreover, if $\rho=4$, then

$$
\Sigma^{2}=\pi\left(\mathbb{S}^{1}\left(\sqrt{\frac{9 \pm \sqrt{41}}{20}}\right) \times \mathbb{S}^{1}\left(\sqrt{\frac{11 \mp \sqrt{41}}{40}}\right) \times \mathbb{S}^{1}\left(\sqrt{\frac{11 \mp \sqrt{41}}{40}}\right)\right) \subset \mathbb{C} P^{2}(4),
$$

where $\pi: \mathbb{S}^{5}(1) \rightarrow \mathbb{C} P^{2}(4)$ is the Hopf fibration.

(c) $\Sigma^{2}$ lies in $\mathbb{C} P^{3}(\rho)$ and

$$
\Sigma^{2}=\gamma_{1} \times \gamma_{2} \subset \mathbb{C} P^{3}(\rho),
$$

where $\gamma_{1}: \mathbb{R} \rightarrow \mathbb{C} P^{2}(\rho) \subset \mathbb{C} P^{3}(\rho)$ is a holomorphic helix of order 4 with curvatures

$$
\kappa_{1}=\sqrt{\frac{7 \rho}{6}}, \quad \kappa_{2}=\frac{1}{2} \sqrt{\frac{5 \rho}{42}}, \quad \kappa_{3}=\frac{3}{2} \sqrt{\frac{\rho}{42}},
$$

and complex torsions

$$
\tau_{12}=-\tau_{34}=\frac{11 \sqrt{14}}{42}, \quad \tau_{23}=-\tau_{14}=\frac{\sqrt{70}}{42}, \quad \tau_{13}=\tau_{24}=0,
$$

and $\gamma_{2}: \mathbb{R} \rightarrow \mathbb{C} P^{3}(\rho)$ is a holomorphic circle with curvature $\kappa=\sqrt{\rho / 2}$ and complex torsion $\tau_{12}=0$. Moreover, the curves $\gamma_{1}$ and $\gamma_{2}$ always exist and are unique up to holomorphic isometries. 
Proof

Let $\Sigma^{2}$ be a complete proper-biharmonic pmc surface with nonnegative Gaussian curvature $K$ and mean curvature vector field $H$ in $\mathbb{C} P^{n}(\rho)$. Let $T$ and $N$ be the tangent and the normal parts of $J H$, respectively. As we have seen in Proposition 4.4, the length of $T$ is constant along the surface. We shall consider two cases: $T=0$ or $T \neq 0$ on $\Sigma^{2}$.

Case I: $T=0$. From Proposition 4.6 we know that $n \geq 3$ and the surface is pseudo-umbilical and totally real with mean curvature $|H|=\sqrt{\rho} / 2$. Consider the Hopf fibration $\pi: \mathbb{S}^{2 n+1}(\rho / 4) \rightarrow \mathbb{C} P^{n}(\rho)$ and the horizontal lift $\widetilde{\Sigma}^{2}$ of $\Sigma^{2}$ to $\mathbb{S}^{2 n+1}(\rho / 4)$. Then, from [23, Theorem 1], we have that $\widetilde{\Sigma}^{2}$ is pseudo-umbilical in $\mathbb{S}^{2 n+1}(\rho / 4)$ and has parallel mean curvature vector field. Moreover, its mean curvature is constant and equal to $\sqrt{\rho} / 2$. Next, using the relation between the bitension fields of the immersions $i: \Sigma^{2} \rightarrow \mathbb{C} P^{n}(\rho)$ and $\widetilde{i}: \widetilde{\Sigma}^{2} \rightarrow \mathbb{S}^{2 n+1}(\rho / 4)$, given in [12, Theorem 3.3], together with $(J H)^{\top}=T=0$, we get that $\Sigma^{2}$ is properbiharmonic if and only if $\widetilde{\Sigma}^{2}$ is proper biharmonic. We apply Theorem 4.9 to conclude that $\widetilde{\Sigma}^{2}$ is a complete minimal surface in a small hypersphere $\mathbb{S}^{2 n}(\rho / 2) \subset$ $\mathbb{S}^{2 n+1}(\rho / 4)$.

Case II: $T \neq 0$. In this case $\Sigma^{2}$ is totally real and, from Proposition 4.7, flat. From the same Proposition 4.7 we also know that $\nabla A_{H}=0$, which means that the eigenfunctions of $A_{H}$ are actually constants. Since $\Sigma^{2}$ being pseudo-umbilical implies that $T=0$, it follows that the surface does not have umbilical points.

Now, let $U$ be a normal vector field orthogonal to $H$ and to $J\left(T \Sigma^{2}\right)$. Then, it is easy to see that $\langle\bar{R}(X, Y) H, U\rangle=0$, and from the Ricci equation (4.4), we get that $\left[A_{H}, A_{U}\right]=0$. Since $H$ is not umbilical, this implies that $A_{H}$ and $A_{U}$ can be simultaneously diagonalized.

On the other hand, the first equation of (4.1) shows that $\operatorname{trace}\left(A_{H} A_{U}\right)=0$ and, therefore, that $A_{U}=0$.

Let us consider the global orthonormal frame field $\left\{E_{1}=T /|T|, E_{2}\right\}$ on the surface. We know, from Remark 4.5, that $\nabla E_{1}=0$ and then $\nabla E_{2}=0$.

Next, if $|T|=|H|$, that is, if $J H$ is a tangent vector field, then we consider the subbundle $L=\operatorname{span}\left\{J E_{1}, J E_{2}\right\}$ of the normal bundle. Since $J H$ is tangent, we get that $H \in L$, and therefore, for any normal vector field $U \perp L$, we have that $A_{U}=0$, which means that $\operatorname{Im} \sigma \subset L$. It is also easy to see that $\operatorname{dim}\left(T \Sigma^{2} \oplus L\right)=4$ and $J\left(T \Sigma^{2} \oplus L\right)=T \Sigma^{2} \oplus L$, which implies that $\bar{R}(X, Y) Z \in L$, for all vector fields $X, Y, Z \in T \Sigma^{2} \oplus L$; that is, $T \Sigma^{2} \oplus L$ is invariant by $\bar{R}$. In the following, we shall prove that $L$ is parallel; that is, if $U$ is a normal vector field orthogonal to $L$, then $U$ is also orthogonal to $\nabla^{\perp} L$. Indeed, for all tangent vector fields $X$ and $Y$, we obtain that

$$
\left\langle\nabla_{X}^{\perp} J Y, U\right\rangle=\left\langle\bar{\nabla}_{X} J Y, U\right\rangle=\left\langle J \bar{\nabla}_{X} Y, U\right\rangle=\left\langle J \nabla_{X} Y+J \sigma(X, Y), U\right\rangle=0,
$$

since $\operatorname{Im} \sigma \subset L$ and $J\left(T \Sigma^{2} \oplus L\right)=T \Sigma^{2} \oplus L$. Therefore, since $\bar{\nabla} \bar{R}=0$, we can use [10, Theorem 2] to show that there exists a 4-dimensional totally geodesic submanifold of $\mathbb{C} P^{n}(\rho)$ such that $\Sigma^{2}$ lies in this submanifold. Since $J\left(T \Sigma^{2} \oplus L\right)=$ $T \Sigma^{2} \oplus L$, we get that $\Sigma^{2}$ is a complete Lagrangian proper-biharmonic pmc surface 
in $\mathbb{C} P^{2}(\rho)$. When $\rho=4$, these surfaces were determined in [24] as follows:

$$
\Sigma^{2}=\pi\left(\mathbb{S}^{1}\left(\sqrt{\frac{9 \pm \sqrt{41}}{20}}\right) \times \mathbb{S}^{1}\left(\sqrt{\frac{11 \mp \sqrt{41}}{40}}\right) \times \mathbb{S}^{1}\left(\sqrt{\frac{11 \mp \sqrt{41}}{40}}\right)\right) \subset \mathbb{C} P^{2}(4) .
$$

Now, assume that $|T|<|H|$. Since $\Sigma^{2}$ is totally real, we can consider the following local normal orthonormal frame field:

$$
\left\{E_{3}=J E_{1}, E_{4}=J E_{2}, E_{5}=\frac{1}{|N|} J N, E_{6}=\frac{1}{|N|} N, E_{7}, \ldots, E_{2 n}\right\},
$$

where $E_{3}, E_{4}, E_{5}$, and $E_{6}$ are globally defined. It can be easily verified that $H$ is orthogonal to $E_{4}, E_{6}$, and $E_{\alpha}$, where $\alpha \in\{7, \ldots, 2 n\}$, and, therefore, that

$$
H=-|T| E_{3}-|N| E_{5} .
$$

All vector fields $E_{\alpha}, \alpha \geq 7$, are orthogonal to $H$ and to $J\left(T \Sigma^{2}\right)$, which means that $A_{\alpha}=0, \alpha \geq 7$, and therefore, $\operatorname{Im} \sigma \subset L=\operatorname{span}\left\{E_{3}, E_{4}, E_{5}, E_{6}\right\}$. Moreover, the bundle $T \Sigma^{2} \oplus L$ is invariant by $J$ and by $\bar{R}$. Let $U$ be a normal vector field, orthogonal to $L$. Using the facts that $\nabla T=0, \operatorname{Im} \sigma \subset L=\operatorname{span}\left\{E_{3}, E_{4}, E_{5}, E_{6}\right\}$, and $J\left(T \Sigma^{2} \oplus L\right)=T \Sigma^{2} \oplus L$, one obtains that

$$
\begin{aligned}
\left\langle\nabla_{X}^{\perp} J Y, U\right\rangle & =\left\langle\bar{\nabla}_{X} J Y, U\right\rangle=\left\langle J \bar{\nabla}_{X} Y, U\right\rangle=\left\langle J \nabla_{X} Y+J \sigma(X, Y), U\right\rangle=0, \\
\left\langle\nabla_{X}^{\perp} N, U\right\rangle & =\left\langle\bar{\nabla}_{X}(J H-T), U\right\rangle=\left\langle-J A_{H} X-\sigma(X, T), U\right\rangle=0
\end{aligned}
$$

and

$$
\left\langle\nabla_{X}^{\perp} J N, U\right\rangle=\left\langle\bar{\nabla}_{X}(-H-J T), U\right\rangle=\langle-J \sigma(X, T), U\rangle=0,
$$

which show that $L$ is parallel. We again use [10, Theorem 2] to conclude that $\Sigma^{2}$ lies in $\mathbb{C} P^{3}(\rho)$.

In the following we shall determine the shape operators $A_{3}, A_{4}, A_{5}$, and $A_{6}$. First, since $N$ is orthogonal to $H$ and to $J\left(T \Sigma^{2}\right)$, we have that $A_{6}=0$. Next, since $\Sigma^{2}$ is totally real, we have that

$$
\langle\sigma(X, Y), J Z\rangle=\langle\sigma(X, Z), J Y\rangle, \quad \forall X, Y, Z \in C\left(T \Sigma^{2}\right),
$$

and using this property together with

$$
\operatorname{trace} A_{3}=2\left\langle E_{3}, H\right\rangle=-2|T| \quad \text { and } \quad \operatorname{trace} A_{4}=2\left\langle E_{4}, H\right\rangle=0,
$$

we see that $A_{3}$ and $A_{4}$ can be written as

$$
A_{3}=\left(\begin{array}{cc}
a-|T| & b \\
b & -a-|T|
\end{array}\right) \quad \text { and } \quad A_{4}=\left(\begin{array}{cc}
b & -a-|T| \\
-a-|T| & -b
\end{array}\right) .
$$

As for $A_{5}$, we have that trace $A_{5}=2\left\langle E_{5}, H\right\rangle=-2|N|$ and then

$$
A_{5}=\left(\begin{array}{cc}
c-|N| & d \\
d & -c-|N|
\end{array}\right) .
$$

Taking into account that $E_{5}$ is orthogonal to $J\left(T \Sigma^{2}\right)$ we obtain that $\langle\bar{R}(X$, $\left.Y) H, E_{5}\right\rangle=0$, and from the Ricci equation (4.4), we see that $\left[A_{H}, A_{5}\right]=0$. Then, from (4.7), we see that $\left[A_{3}, A_{5}\right]=0$. After a straightforward computation, we get 
that

$$
a d=b c .
$$

Next, we have $\left\langle\bar{R}\left(E_{2}, E_{1}\right) H, J E_{2}\right\rangle=-(\rho / 4)|T|$ and then, again using the Ricci formula (4.4),

$$
\left\langle\left[A_{H}, A_{4}\right] E_{1}, E_{2}\right\rangle=\left\langle\bar{R}\left(E_{2}, E_{1}\right) H, J E_{2}\right\rangle=-\frac{\rho}{4}|T|,
$$

which can be written as

$$
b(b|T|+d|N|)+(a+|T|)(a|T|+c|N|)=\frac{\rho}{8}|T| .
$$

Since $\Sigma^{2}$ is proper biharmonic, from the first equation of (4.1), taking into account that $E_{4}$ is orthogonal to $H$ and to $E_{3}$, we see that $\operatorname{trace}\left(A_{H} A_{4}\right)=0$, which, using (4.8), gives that

$$
b|T|+d|N|=0 .
$$

Assume now that there exists a point $p \in \Sigma^{2}$ such that $b \neq 0$ or $d \neq 0$ at $p$. Then, from (4.8), (4.9), and (4.10), we obtain that $|T|=0$ at $p$, which is a contradiction. Therefore, from (4.10), it follows that $b=d=0$ on $\Sigma^{2}$, and then (4.9) becomes

$$
(a+|T|)(a|T|+c|N|)=\frac{\rho}{8}|T| .
$$

Finally, again using the first equation of (4.1), we have that

$$
\operatorname{trace}\left(A_{H} A_{3}\right)=-\frac{5 \rho}{4}|T| \quad \text { and } \quad \operatorname{trace}\left(A_{H} A_{5}\right)=-\frac{\rho}{2}|N|,
$$

or equivalently,

$$
a(a|T|+c|N|)=\frac{\left(5 \rho-8|H|^{2}\right)|T|}{8}
$$

and

$$
c(a|T|+c|N|)=\frac{\left(\rho-4|H|^{2}\right)|N|}{4},
$$

respectively. From (4.11), (4.12), and (4.13) one obtains that

$$
a=\frac{\left(5 \rho-8|H|^{2}\right)|T|}{4\left(2|H|^{2}-\rho\right)}, \quad c=\frac{\left(\rho-4|H|^{2}\right)|N|}{2\left(2|H|^{2}-\rho\right)},
$$

and

$$
16|H|^{4}-10 \rho|H|^{2}-3 \rho|T|^{2}+2 \rho^{2}=0 .
$$

The surface $\Sigma^{2}$ is flat, and therefore, by its Gauss equation from (3.6) in $\mathbb{C} P^{n}(\rho)$, it follows that

$$
0=K=\frac{\rho}{4}+\sum_{\alpha=3}^{5} \operatorname{det} A_{\alpha},
$$

which, together with (4.14), gives that

$$
16|H|^{4}+4 \rho|H|^{2}-48|T|^{2}|H|^{2}+22 \rho|T|^{2}-4 \rho^{2}=0 .
$$


From (4.15) and (4.16) we obtain that $|H|^{2}=\rho / 3,|T|^{2}=4 \rho / 27$, and $|N|^{2}=$ $5 \rho / 27$. Hence, the shape operator $A$ is given by

$$
\begin{aligned}
& A_{3}=\frac{1}{2} \sqrt{\frac{\rho}{3}}\left(\begin{array}{cc}
-\frac{11}{3} & 0 \\
0 & 1
\end{array}\right), \quad A_{4}=\frac{1}{2} \sqrt{\frac{\rho}{3}}\left(\begin{array}{ll}
0 & 1 \\
1 & 0
\end{array}\right), \\
& A_{5}=-\frac{1}{2} \sqrt{\frac{5 \rho}{3}}\left(\begin{array}{cc}
-\frac{1}{3} & 0 \\
0 & 1
\end{array}\right) .
\end{aligned}
$$

Now, since $E_{1}$ and $E_{2}$ are parallel, they determine two distributions which are mutually orthogonal, smooth, involutive, and parallel. Therefore, from the de Rham decomposition theorem and by taking into account that the surface is complete and using its universal cover if necessary, it follows that $\Sigma^{2}$ is the standard product $\gamma_{1} \times \gamma_{2}$, where $\gamma_{k}: \mathbb{R} \rightarrow \mathbb{C} P^{3}(\rho), k \in\{1,2\}$, are integral curves of $E_{1}$ and $E_{2}$, respectively, parameterized by arc length, that is, $\gamma_{1}^{\prime}=E_{1}$ and $\gamma_{2}^{\prime}=E_{2}$ (see [17]). In the following, we shall determine these curves in terms of their curvatures and complex torsions.

Let us denote by $\kappa_{i}, 1 \leq i<6$, the curvatures of $\gamma_{1}$ and by $\left\{X_{j}^{1}\right\}, 1 \leq j<7$, its Frenet frame field. Using (4.17), we first have that

$$
\bar{\nabla}_{E_{1}} E_{1}=\sigma\left(E_{1}, E_{1}\right)=-\frac{11}{6} \sqrt{\frac{\rho}{3}} E_{3}-\frac{1}{6} \sqrt{\frac{5 \rho}{3}} E_{5},
$$

and then, the first Frenet equation of $\gamma_{1}$ gives that

$$
\kappa_{1}=\sqrt{\frac{7 \rho}{6}} \quad \text { and } \quad X_{2}^{1}=-\frac{11 \sqrt{14}}{42} E_{3}-\frac{\sqrt{70}}{42} E_{5} .
$$

Next, since $\nabla E_{1}=\nabla E_{2}=0$ and $0=\nabla^{\perp} H=-|T| \nabla^{\perp} E_{3}-|N| \nabla^{\perp} E_{5}$, we get that

$$
\left\langle\nabla_{E_{1}}^{\perp} E_{3}, E_{4}\right\rangle=0, \quad\left\langle\nabla_{E_{1}}^{\perp} E_{3}, E_{5}\right\rangle=0
$$

and

$$
\begin{aligned}
\left\langle\nabla_{E_{1}}^{\perp} E_{3}, E_{6}\right\rangle & =\frac{1}{|N|}\left\langle\bar{\nabla}_{E_{1}} J E_{1}, J H-T\right\rangle=\frac{1}{|N|}\left(\left\langle A_{H} E_{1}, E_{1}\right\rangle+|T|\left\langle\bar{\nabla}_{E_{1}} E_{1}, E_{3}\right\rangle\right) \\
& =-\left\langle A_{5} E_{1}, E_{1}\right\rangle=\frac{1}{6} \sqrt{\frac{5 \rho}{3}} .
\end{aligned}
$$

It follows that $\nabla_{E_{1}}^{\perp} E_{3}=(1 / 6) \sqrt{5 \rho / 3} E_{6}$. In the same way, one obtains that $\nabla_{E_{1}}^{\perp} E_{5}=(-1 / 3) \sqrt{\rho / 3} E_{6}$. Thus, after a straightforward computation,

$$
\bar{\nabla}_{E_{1}} X_{2}^{1}=-\kappa_{1} E_{1}-\frac{1}{2} \sqrt{\frac{5 \rho}{42}} E_{6}
$$

which means that

$$
\kappa_{2}=\frac{1}{2} \sqrt{\frac{5 \rho}{42}} \quad \text { and } \quad X_{3}^{1}=-E_{6}
$$

It follows that

$$
\bar{\nabla}_{E_{1}} X_{3}^{1}=\frac{1}{6} \sqrt{\frac{5 \rho}{3}} E_{3}-\frac{1}{3} \sqrt{\frac{\rho}{3}} E_{5}
$$


and then

$$
\kappa_{3}=\frac{3}{2} \sqrt{\frac{\rho}{42}} \quad \text { and } \quad X_{4}^{1}=\frac{\sqrt{70}}{42} E_{3}-\frac{11 \sqrt{14}}{42} E_{5} .
$$

Finally, we get that $\bar{\nabla}_{E_{1}} X_{4}^{1}=-\kappa_{3} X_{3}^{1}$, and therefore, $\gamma_{1}$ is a helix of osculating order 4. A simple computation gives its complex torsions

$$
\tau_{12}=-\tau_{34}=\frac{11 \sqrt{14}}{42}, \quad \tau_{23}=-\tau_{14}=\frac{\sqrt{70}}{42}, \quad \tau_{13}=\tau_{24}=0 .
$$

Hence, $\gamma_{1}$ is a holomorphic helix of order 4 .

Consider now the subbundle $L=\operatorname{span}\left\{E_{3}, E_{5}, E_{6}\right\}$ in the normal bundle of $\gamma_{1}$. It is easy to see that $L$ is parallel and $T \gamma_{1} \oplus L$ is invariant by $J$ and $\bar{R}$. Then, since $X_{2}^{1} \in L$, we apply [10, Theorem 2] to conclude that $\gamma_{1}$ lies in $\mathbb{C} P^{2}(\rho)$. Moreover, it can be easily verified that $\gamma_{1}$ is of class $I_{3}$.

For the curve $\gamma_{2}$ we have that

$$
\bar{\nabla}_{E_{2}} E_{2}=\sigma\left(E_{2}, E_{2}\right)=\frac{1}{2} \sqrt{\frac{\rho}{3}} E_{3}-\frac{1}{2} \sqrt{\frac{5 \rho}{3}} E_{5},
$$

and then its first curvature is $\kappa=\sqrt{\rho / 2}$ and $X_{2}^{2}=(\sqrt{6} / 6) E_{3}-(\sqrt{30} / 6) E_{5}$. It can be easily verified that $\nabla_{E_{2}}^{\perp} E_{3}=\nabla_{E_{2}}^{\perp} E_{5}=0$, and then one obtains that $\bar{\nabla}_{E_{2}} X_{2}^{2}=$ $-\kappa E_{2}$. Therefore, the curve $\gamma_{2}$ is a holomorphic circle in $\mathbb{C} P^{3}(\rho)$ with curvature $\kappa=\sqrt{\rho / 2}$ and complex torsion $\tau_{12}=0$. Then, we use Theorems 2.2 and 2.3 to conclude.

\section{REMARK 4.11}

By working in the same way as in the case when $\rho=4$, considered in [24], the result in Theorem $4.10\left(\right.$ b) can be extended to surfaces in $\mathbb{C} P^{n}(\rho)$. However, for the sake of simplicity we present here only this particular case.

Acknowledgments. The authors wish to thank Cezar Oniciuc for many useful comments and helpful discussions. The first author would like to thank the Department of Mathematics of the Federal University of Bahia in Salvador for providing a very stimulative work environment during the preparation of this article.

\section{References}

[1] H. Alencar, M. do Carmo, and R. Tribuzy, A Hopf theorem for ambient spaces of dimensions higher than three, J. Differential Geom. 84 (2010), 1-17. MR 2629507.

[2] A. Balmuş, S. Montaldo, and C. Oniciuc, Classification results for biharmonic submanifolds in spheres, Israel J. Math. 168 (2008), 201-220. MR 2448058. DOI 10.1007/s11856-008-1064-4.

[3] B Biharmonic PNMC submanifolds in spheres, Ark. Mat. 51 (2013), 197-221. MR 3090194. DOI 10.1007/s11512-012-0169-5. 
[4] A. Balmuş and C. Oniciuc, Biharmonic submanifolds with parallel mean curvature vector field in spheres, J. Math. Anal. Appl. 386 (2012), 619-630. MR 2834772. DOI 10.1016/j.jmaa.2011.08.019.

[5] M. H. Batista da Silva, Simons type equation in $\mathbb{S}^{2} \times \mathbb{R}$ and $\mathbb{H}^{2} \times \mathbb{R}$ and applications, Ann. Inst. Fourier (Grenoble) 61 (2011), 1299-1322. MR 2951494. DOI 10.5802/aif.2641.

[6] B.-Y. Chen, A report on submanifolds of finite type, Soochow J. Math. 22 (1996), 117-337. MR 1391469.

[7] B.-Y. Chen and K. Ogiue, On totally real submanifolds, Trans. Amer. Math. Soc. 193 (1974), 257-266. MR 0346708.

[8] S. Y. Cheng and S. T. Yau, Hypersurfaces with constant scalar curvature, Math. Ann. 225 (1977), 195-204. MR 0431043.

[9] J. Eells, Jr. and J. H. Sampson, Harmonic mappings of Riemannian manifolds, Amer. J. Math. 86 (1964), 109-160. MR 0164306.

[10] J.-H. Eschenburg and R. Tribuzy, Existence and uniqueness of maps into affine homogeneous spaces, Rend. Sem. Mat. Univ. Padova 89 (1993), 11-18. MR 1229038.

[11] D. Fetcu, Surfaces with parallel mean curvature vector in complex space forms, J. Differential Geom. 91 (2012), 215-232. MR 2971287.

[12] D. Fetcu, E. Loubeau, S. Montaldo, and C. Oniciuc, Biharmonic submanifolds of $\mathbb{C} P^{n}$, Math. Z. 266 (2010), 505-531. MR 2719418.

DOI 10.1007/s00209-009-0582-z.

[13] D. Fetcu and C. Oniciuc, Biharmonic integral $\mathcal{C}$-parallel submanifolds in 7-dimensional Sasakian space forms, Tohoku Math. J. (2) 64 (2012), 195-222. MR 2948819. DOI 10.2748/tmj/1341249371.

[14] D. Fetcu, C. Oniciuc, and H. Rosenberg, Biharmonic submanifolds with parallel mean curvature in $\mathbb{S}^{n} \times \mathbb{R}$, J. Geom. Anal. 23 (2013), 2158-2176. MR 3107694. DOI 10.1007/s12220-012-9323-3.

[15] A. Huber, On subharmonic functions and differential geometry in the large, Comment. Math. Helv. 32 (1957), 13-72. MR 0094452.

[16] G. Y. Jiang, 2-harmonic maps and their first and second variational formulas, Chinese Ann. Math. Ser. A 7 (1986), 389-402. MR 0886529.

[17] S. Kobayashi and K. Nomizu, Foundations of Differential Geometry, I, Interscience, New York, 1963. MR 0152974.

[18] E. Loubeau and C. Oniciuc, Biharmonic surfaces of constant mean curvature, Pacific J. Math. 271 (2014), 213-230. MR 3259766.

DOI 10.2140/pjm.2014.271.213.

[19] S. Maeda and T. Adachi, Holomorphic helices in a complex space form, Proc. Amer. Math. Soc. 125 (1997), 1197-1202. MR 1353391. DOI 10.1090/S0002-9939-97-03627-7.

[20] S. Maeda and Y. Ohnita, Helical geodesic immersions into complex space forms, Geom. Dedicata 30 (1989), 93-114. MR 0995941. DOI 10.1007/BF02424315. 
[21] S. Maeta and H. Urakawa, Biharmonic Lagrangian submanifolds in Kähler manifolds, Glasg. Math. J. 55 (2013), 465-480. MR 3040876.

DOI 10.1017/S0017089512000730.

[22] Y.-L. Ou and Z.-P. Wang, Constant mean curvature and totally umbilical biharmonic surfaces in 3-dimensional geometries, J. Geom. Phys. 61 (2011), 1845-1853. MR 2822453. DOI 10.1016/j.geomphys.2011.04.008.

[23] H. Reckziegel, "Horizontal lifts of isometric immersions into the bundle space of a pseudo-Riemannian submersion" in Global Differential Geometry and Global Analysis 1984 (Berlin, 1984), Lecture Notes in Math. 1156, Springer, Berlin, 1985, 264-279. MR 0824074. DOI 10.1007/BFb0075098.

[24] T. Sasahara, Biharmonic Lagrangian surfaces of constant mean curvature in complex space forms, Glasg. Math. J. 49 (2007), 497-507. MR 2371514. DOI 10.1017/S0017089507003886.

[25] N. Sato, Totally real submanifolds of a complex space form with nonzero parallel mean curvature vector, Yokohama Math. J. 44 (1997), 1-4. MR 1453345.

[26] J. Simons, Minimal varieties in Riemannian manifolds, Ann. of Math. (2) 88 (1968), 62-105. MR 0233295.

[27] W. Zhang, New examples of biharmonic submanifolds in $\mathbb{C} P^{n}$ and $\mathbb{S}^{2 n+1}$, An. Ştiinţ. Univ. Al. I. Cuza Iaşi. Mat. (N.S.) 57 (2011), 207-218. MR 2985674. DOI 10.2478/v10157-010-0046-0.

Fetcu: Department of Mathematics and Informatics, Gheorghe Asachi Technical University of Iasi, Bulevardul Carol I, 11, 700506 Iasi, Romania; and Department of Mathematics, Federal University of Bahia, Avenida Adhemar de Barros s/n, 40170-110 Salvador, Bahia, Brazil; dfetcu@math.tuiasi.ro

Pinheiro: Department of Mathematics, Federal University of Bahia, Avenida Adhemar de Barros s/n, 40170-110 Salvador, Bahia, Brazil; anapinhe@ufba.br 